\title{
DepQBF 6.0: A Search-Based QBF Solver Beyond Traditional QCDCL ${ }^{\star}$
}

\author{
Florian Lonsing and Uwe Egly \\ Knowledge-Based Systems Group, Vienna University of Technology, Austria \\ firstname.lastname@tuwien.ac.at
}

\begin{abstract}
We present the latest major release version 6.0 of the quantified Boolean formula ( $Q B F$ ) solver DepQBF, which is based on $Q C D C L$. QCDCL is an extension of the conflict-driven clause learning (CDCL) paradigm implemented in state of the art propositional satisfiability (SAT) solvers. The Q-resolution calculus (QRES) is a QBF proof system which underlies QCDCL. QCDCL solvers can produce QRES proofs of QBFs in prenex conjunctive normal form (PCNF) as a byproduct of the solving process. In contrast to traditional QCDCL based on QRES, DepQBF 6.0 implements a variant of QCDCL which is based on a generalization of QRES. This generalization is due to a set of additional axioms and leaves the original Q-resolution rules unchanged. The generalization of QRES enables QCDCL to potentially produce exponentially shorter proofs than the traditional variant. We present an overview of the features implemented in DepQBF and report on experimental results which demonstrate the effectiveness of generalized QRES in QCDCL.
\end{abstract}

\section{Introduction}

Propositional satisfiability (SAT) solvers based on conflict-driven clause learning (CDCL) [43 implement a combination of the DPLL algorithm [1] and propositional resolution [40] to derive learned clauses from a CNF to be solved.

CDCL has been extended to solve quantified Boolean formulas (QBFs) 20], resulting in the $Q C D C L$ approach 14/2448. The logic of QBFs allows for explicit universal and existential quantification of propositional variables. As a consequence, the satisfiability problem of QBFs is PSPACE-complete.

In contrast to SAT solving, where CDCL is the dominant solving paradigm in practice, QCDCL is complemented by variable expansion [16]. This approach successively eliminates variables from a QBF until it reduces to either true or false. Many modern solvers (e.g. [17/19/39) implement expansion by counter-example guided abstraction refinement (CEGAR) [10].

The Q-resolution calculus (QRES) [14|21|24]48] is a QBF proof system that underlies QCDCL in a way that is analogous to propositional resolution in CDCL.

\footnotetext{
* Supported by the Austrian Science Fund (FWF) under grant S11409-N23. This article will appear in the proceedings of the 26th International Conference on Automated Deduction (CADE-26), LNCS, Springer, 2017.
} 
The empty clause is derivable from a PCNF $\psi$ by QRES iff $\psi$ is unsatisfiable. According to QBF proof complexity, there is an exponential separation between the sizes of proofs that variable expansion and Q-resolution can produce for certain QBFs [5]18. This theoretical result suggests to combine such orthogonal proof systems in QBF solvers to leverage their individual strengths.

As a first step towards a solver framework that allows for the combination of QBF proof systems in a systematic way, we present the latest major release version 6.0 of the QCDCL solver DepQBF 1 In contrast to traditional QCDCL based on QRES [14|21|24|48], DepQBF 6.0 implements a variant of QCDCL that relies on a generalization of QRES. This generalization is due to a set of new axioms added to QRES 31. In practice, derivations made by the added axioms in QCDCL are based on arbitrary QBF proof systems. As a consequence, when applying proof systems that are orthogonal to Q-resolution, the generalization of QRES via the new axioms enables QCDCL as implemented in DepQBF 6.0 to potentially produce exponentially shorter proofs than traditional QCDCL.

We report on experiments where we compare DepQBF 6.0 to state of the art QBF solvers. Experimental results demonstrate the effectiveness of generalized QRES in QCDCL. Additionally, we briefly summarize the evolution of DepQBF since the first version 0.1 26. We relate the features that were added to the different versions of DepQBF over time to the enhanced variant of QCDCL implemented in DepQBF 6.0.

\section{Preliminaries}

A QBF $\psi:=\Pi . \phi$ in prenex conjunctive normal form (PCNF) consists of a quantifier prefix $\Pi:=Q_{1} X_{1} \ldots Q_{n} X_{n}$ and a CNF $\phi$ not containing tautological clauses. The CNF $\phi$ is defined over the propositional variables $X_{1} \cup \ldots \cup X_{n}$ that appear in $\Pi$. The variable sets $X_{i}$ are pairwise disjoint and $Q_{i} \neq Q_{i+1}$ for $Q_{i} \in\{\forall, \exists\}$. QBFs $\psi:=\Pi$. $\phi$ in prenex disjunctive normal form (PDNF) are defined analogously to PCNFs, where $\phi$ is a DNF consisting of cubes. A cube is a conjunction of literals. The quantifier $\mathrm{Q}(\Pi, l)$ of a literal $l$ is $Q_{i}$ if the variable $\operatorname{var}(l)$ of $l$ appears in $X_{i}$. If $\mathrm{Q}(\Pi, l)=Q_{i}$ and $\mathrm{Q}(\Pi, k)=Q_{j}$, then $l \leq_{\Pi} k$ iff $i \leq j$.

An assignment $A$ maps variables of a $\mathrm{QBF} \Pi$. $\phi$ to truth values true $(\top)$ and false $(\perp)$. We represent $A=\left\{l_{1}, \ldots, l_{n}\right\}$ as a set of literals such that if a variable $x$ is assigned true (false) then $l_{i} \in A$ with $l_{i}=x\left(l_{i}=\bar{x}\right)$, where $\bar{x}$ is the negation of $x$. Further, $\operatorname{var}\left(l_{i}\right) \neq \operatorname{var}\left(l_{j}\right)$ for any $l_{i}, l_{j} \in A$ with $i \neq j$.

The PCNF $\psi$ under assignment $A$, written as $\psi[A]$, is the PCNF obtained from $\psi$ in which for all $l \in A$, all clauses containing $l$ are removed, all occurrences of $\bar{l}$ are deleted, and $\operatorname{var}(l)$ is removed from the prefix. If the CNF of $\psi[A]$ is empty (respectively, contains the empty clause $\emptyset$ ), then it is satisfied (falsified) by $A$ and $A$ is a satisfying (falsifying) assignment, written as $\psi[A]=\top(\psi[A]=\perp)$. A PDNF $\psi$ under an assignment $A$ and an empty cube are defined in a way dual to PCNFs and empty clauses. A QBF $\Pi . \phi$ with $Q_{1}=\exists\left(Q_{1}=\forall\right)$ is satisfiable iff,

\footnotetext{
${ }^{1}$ DepQBF is licensed under GPLv3: http://lonsing.github.io/depqbf/
} 


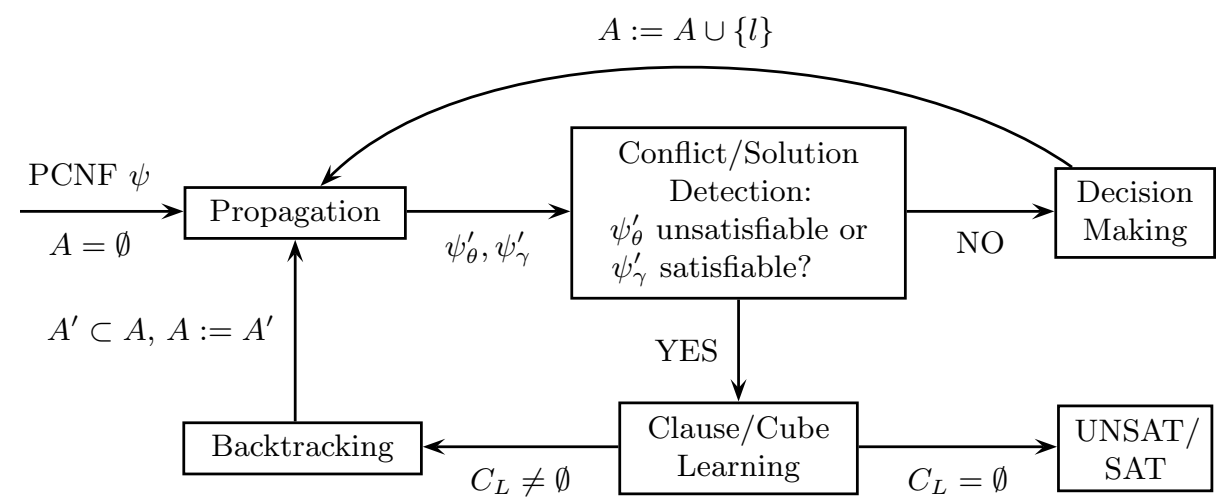

Fig. 1. Workflow of the variant of QCDCL implemented in DepQBF 6.0 that relies on a generalization of the Q-resolution calculus (QRES) (figure adapted from [31]).

for $x \in X_{1}, \Pi . \phi[\{x\}]$ or (and) $\Pi . \phi[\{\bar{x}\}]$ is satisfiable. Two QBFs $\psi$ and $\psi^{\prime}$ are satisfiability-equivalent $\left(\psi \equiv_{\text {sat }} \psi^{\prime}\right)$, iff $\psi$ is satisfiable whenever $\psi^{\prime}$ is satisfiable.

\section{QCDCL and the Generalized Q-Resolution Calculus}

In the following, we present the variant of QCDCL implemented in DepQBF 6.0 that relies on a generalization of the Q-resolution calculus (QRES). We illustrate the workflow of that variant in Fig. 1

In general, QCDCL is based on the successive generation of assignments that guide the application of the inference rules of QRES to derive learned clauses and cubes from a given input PCNF $\psi=\Pi$. $\phi$. Learned cubes are dual to clauses. While learned clauses represent assignments that falsify the CNF $\phi$ of $\psi$, learned cubes represent assignments that satisfy $\phi$. The empty cube is derivable from a PCNF $\psi$ by QRES iff $\psi$ is satisfiable. Based on our presentation of the rules of QRES we illustrate the differences between traditional QCDCL and the variant implemented in DepQBF 6.0.

A QCDCL solver maintains a PCNF $\theta=\Pi \cdot \theta^{\prime}$ (PDNF $\left.\gamma=\Pi \cdot \gamma^{\prime}\right)$ consisting of a $\mathrm{CNF} \theta^{\prime}\left(\mathrm{DNF} \gamma^{\prime}\right)$ of learned clauses (cubes). The clauses in $\theta$ are added conjunctively to $\psi$ to obtain $\psi_{\theta}=\Pi .\left(\phi \wedge\left(\bigwedge_{C \in \theta^{\prime}} C\right)\right)$, and the cubes in $\gamma$ are added disjunctively to $\psi$ to obtain $\psi_{\gamma}=\Pi \cdot\left(\phi \vee\left(\bigvee_{C \in \gamma^{\prime}} C\right)\right)$. It holds that $\psi \equiv_{s a t} \psi_{\theta}$ and $\psi \equiv_{\text {sat }} \psi_{\gamma}$. Initially the current assignment $A$, the PCNF $\theta$, and PDNF $\gamma$ are empty. We use the notation $C, C^{\prime}$, and $C_{L}$ for both clauses and cubes.

During propagation, the formulas $\psi_{\theta}$ and $\psi_{\gamma}$ are first simplified under the current assignment $A$ by computing $\psi_{\theta}[A]$ and $\psi_{\gamma}[A]$. Then universal and existential reduction is applied to $\psi_{\theta}[A]$ and to $\psi_{\gamma}[A]$ based on the following inference rule. 
Definition 1 (Reduction [14|21,24,48]). Let $\psi=\Pi$. $\phi$ be a PCNF.

$$
\begin{aligned}
& \text { (1) } C \text { is a clause, } \mathrm{Q}(\Pi, l)=\forall \text {, } \\
& C \cup\{l\} \quad l^{\prime}<_{\Pi} l \text { for all } l^{\prime} \in C \text { with } \mathrm{Q}\left(\Pi, l^{\prime}\right)=\exists \text { or } \\
& \text { (2) } C \text { is a cube, } \mathrm{Q}(\Pi, l)=\exists \text {, } \\
& l^{\prime}<_{\Pi} l \text { for all } l^{\prime} \in C \text { with } \mathrm{Q}\left(\Pi, l^{\prime}\right)=\forall
\end{aligned}
$$

Universal (existential) reduction of clauses (cubes) by rule red eliminates trailing universal (existential) literals from a clause (cube) with respect to the linear quantifier ordering in the prefix of the PCNF $\psi$. We write $U R(C)=C^{\prime}$ $\left(E R(C)=C^{\prime}\right)$ to denote the clause (cube) $C^{\prime}$ resulting from clause (cube) $C$ by fully reducing universal (existential) literals.

Let $\psi_{\theta}^{\prime}$ and $\psi_{\gamma}^{\prime}$ denote the formulas obtained by applying universal (existential) reduction to all the clauses (cubes) in $\psi_{\theta}[A]\left(\psi_{\gamma}[A]\right)$ until saturation. New assignments are generated by unit literal detection with respect to $\psi_{\theta}^{\prime}$ and $\psi_{\gamma}^{\prime}$. If a PCNF (PDNF) $\psi$ contains a unit clause (cube) $C=(l)$, where $\mathrm{Q}(\Pi, l)=\exists$ $(\mathrm{Q}(\Pi, l)=\forall)$, then literal $l$ is unit and $\psi \equiv_{\text {sat }} \psi\left[A^{\prime}\right]$ where $A^{\prime}=\{l\}\left(A^{\prime}=\{\bar{l}\}\right)$. Assignment $A$ is extended by assignments $A^{\prime}$ derived from unit clauses (cubes) in $\psi_{\theta}^{\prime}\left(\psi_{\gamma}^{\prime}\right)$. For every unit clause (cube) $C^{\prime} \in \psi_{\theta}^{\prime}\left(C^{\prime} \in \psi_{\gamma}^{\prime}\right)$ with $C^{\prime}=(l)$, the corresponding assignment $A^{\prime}:=\{l\}\left(A^{\prime}:=\{\bar{l}\}\right)$ is recorded.

After propagation, in conflict/solution detection it is checked whether $\psi_{\theta}^{\prime}$ is unsatisfiable or whether $\psi_{\gamma}^{\prime}$ is satisfiable (only one of the two cases can occur). To this end, incomplete methods are applied. In traditional QCDCL, for example, it is syntactically checked if the current assignment $A$ is falsifying or satisfying, i.e., whether $\psi_{\theta}^{\prime}$ contains the empty clause (i.e., $\psi_{\theta}^{\prime}=\perp$ ) or whether $\psi_{\gamma}^{\prime}$ contains the empty cube (i.e., $\psi_{\gamma}^{\prime}=\top$ ). In DepQBF 6.0, we extend these incomplete syntactic checks to incomplete semantic checks based on arbitrary QBF decision procedures (proof systems) that are applied to $\psi_{\theta}^{\prime}$ and $\psi_{\gamma}^{\prime}$ in a resource bounded way.

If neither $\psi_{\theta}^{\prime}$ is found unsatisfiable nor $\psi_{\gamma}^{\prime}$ is found satisfiable by the incomplete satisfiability checks, then in decision making $A$ is extended by heuristically assigning some decision variable $x$ from the leftmost quantifier block of $\psi[A]$ $(A:=A \cup\{l\}$ where $\operatorname{var}(l)=x)$, and propagation continues. Assignments by decision making must follow the prefix ordering of $\psi$, in contrast to assignments by propagation (unit literals), which results in assignments of the following kind.

Definition 2 (QCDCL assignment [25]). Assignments generated by decision making and propagation in QCDCL are called QCDCL assignments.

If $\psi_{\theta}^{\prime}\left(\psi_{\gamma}^{\prime}\right)$ is found unsatisfiable (satisfiable) in conflict/solution detection then a learned clause (cube) is derived using QRES depending on the incomplete satisfiability checks. In traditional QCDCL, conflict/solution detection relies only on falsifying or satisfying assignments. If $\psi_{\theta}^{\prime}=\perp$ then $\psi_{\theta}^{\prime}$ contains an empty clause $C^{\prime}=\emptyset$ such that there is a clause $C \in \psi_{\theta}$ with $C^{\prime}=U R(C[A])$. Clause $C$ is the falsified clause with respect to assignment $A$. If $C$ appears in the given $\mathrm{PCNF} \psi$ then in traditional QRES it is derived trivially by the following axiom.

Definition 3 (Clause Axiom [14,21,24,48]). Let $\psi=\Pi$. $\phi$ be a PCNF. 
If $\psi_{\theta}^{\prime} \neq \perp$ but $\psi_{\gamma}^{\prime}=\top$ then either (1) $\psi_{\gamma}^{\prime}$ contains an empty learned cube $C^{\prime}=\emptyset$ such that there is a cube $C \in \psi_{\gamma}$ with $C^{\prime}=E R(C[A])$, or (2) $A$ is a satisfying assignment that satisfies all clauses in $\psi_{\gamma}^{\prime}$. For case (2), a cube $C$ is derived by the following axiom of traditional QRES (in either case (1) or (2) cube $C$ is the satisfied cube with respect to $A$ ).

Definition 4 (Cube Axiom $[\mathbf{1 4}, \mathbf{2 1}, \mathbf{2 4}, 48]$ ). Let $\psi=\Pi$. $\phi$ be a PCNF.

$$
\bar{C} \quad A \text { is an assignment, } \psi[A]=\top \text {, and } C=\left(\bigwedge_{l \in A} l\right) \text { is a cube } \quad \text { (cu-init) }
$$

DepQBF 6.0 supports the application of arbitrary (incomplete) QBF decision procedures (proof systems) in conflict/solution detection and thus generalizes the syntactic checks for falsifying and satisfying assignments in traditional QCDCL.

To check the satisfiability of $\psi_{\gamma}^{\prime}$, in DepQBF 6.0 we apply a dynamic variant of blocked clause elimination (QBCE) [25]. This approach was introduced in version 5.0 of DepQBF. QBCE has been presented as a preprocessing technique to eliminate redundant blocked clauses [15|23] from a PCNF. If all clauses in $\psi_{\gamma}^{\prime}$ are satisfied under $A$ or identified as blocked, then $\psi_{\gamma}^{\prime}$ is determined satisfiable. In our implementation applications of QBCE are tightly integrated in the propagation phase via efficient data structures. Clauses that are blocked are temporarily considered as removed from the formula. Hence such clauses cannot be used to detect unit clauses or empty clauses during propagation.

In addition to dynamic QBCE, we implemented incomplete QBF satisfiability checks based on propositional abstractions of $\psi_{\theta}^{\prime}$ and $\psi_{\gamma}^{\prime}$ [31, which are solved using an integrated SAT solver. These abstractions are constructed by treating universally quantified literals in the given $\mathrm{PCNF} \psi$ in a special way. Propositional abstractions and SAT solving leverage the benefits of techniques like trivial truth and trivial falsity presented already in early search-based QBF solvers [9]. Additionally, the power of $Q U$-resolution [47, which is exponentially stronger than Q-resolution [21] but has not been applied systematically in QCDCL, is harnessed to a certain extent (cf. Example 3 in [31).

As a simple way of applying a QBF decision procedure that is incomplete by its nature we integrated the preprocessor Bloqqer 15 in DepQBF 6.0. Preprocessing aims at simplifying a formula within a restricted amount of time but might already solve certain formulas (cf. 33). Among several techniques, Bloqqer applies bounded expansion of universally quantified variables [6] integrating Bloqqer in QCDCL we in fact integrate expansion, a QBF proof system that is orthogonal to Q-resolution 5|18. Due to usability issues, in the follow-up release version 6.02 of DepQBF we replaced Bloqqer by the expansion based QBF solver Nenofex ${ }^{2}$ which is applied in a resource bounded way.

If $\psi_{\theta}^{\prime}\left(\psi_{\gamma}^{\prime}\right)$ is found unsatisfiable (satisfiable) by an incomplete decision procedure but, unlike above, $A$ is neither falsifying nor satisfying, then a clause (cube) is derived by the following generalized axioms of QRES. These axioms are added to QRES and applied in addition to the traditional axioms cl-init and cu-init.

\footnotetext{
2 https://github.com/lonsing/nenofex
} 
Definition 5 (Generalized Axioms [31]). Let $\psi=\Pi$. $\phi$ be a PCNF.

$$
\begin{aligned}
& A \text { is a } Q C D C L \text { assignment, } \psi[A] \text { is unsatisfiable, } \\
& \bar{C} \text { and } C=\left(\bigvee_{l \in A} \bar{l}\right) \text { is a clause } \\
& A \text { is a } Q C D C L \text { assignment, } \psi[A] \text { is satisfiable, } \\
& \bar{C} \text { and } C=\left(\bigwedge_{l \in A} l\right) \text { is a cube }
\end{aligned}
$$

Note that the generalized axioms allow to derive clauses and cubes that cannot be derived by the traditional axioms cl-init and cu-init in general. This is due to the application of arbitrary QBF decision procedures (proof systems) for satisfiability checking in conflict/solution detection or in the side conditions of the axioms, respectively. In the side conditions the satisfiability of the PCNF $\psi[A]$ is checked, in contrast to formulas $\psi_{\theta}^{\prime}$ and $\psi_{\gamma}^{\prime}$ as in conflict/solution detection. This is possible since $\psi_{\theta}^{\prime} \equiv_{s a t} \psi[A]$ and $\psi_{\gamma}^{\prime} \equiv_{s a t} \psi[A]$. The clause (cube) $C$ derived by applying the generalized clause axiom gen-cl-init $(g e n-c u$-init $)$ is the falsified clause (satisfied cube) with respect to $A$.

During clause (cube) learning, a new learned clause (cube) $C_{L}$ is derived by QRES. The falsified clause (satisfied cube) $C$ is the start clause (cube) of a derivation of $C_{L}$. Given $A$, clauses (cubes) which became unit during propagation are systematically resolved based on the following $Q$-resolution rule.

Definition 6 (Q-Resolution [21]). Let $\psi=\Pi$. $\phi$ be a PCNF.

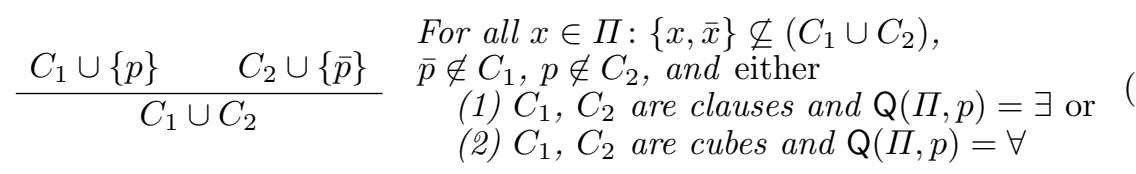

Rule res does not allow the resolvent $\left(C_{1} \cup C_{2}\right)$ to be a tautological clause (contradictory cube) and requires existential (universal) variables as pivots $p$. In general, learning produces a nonempty clause (cube) $C_{L} \neq \emptyset$, which is added to the PCNF $\theta$ (PDNF $\gamma$ ) of learned clauses (cubes), and hence also to $\psi_{\theta}\left(\psi_{\gamma}\right)$.

In backtracking, a certain subassignment $A^{\prime} \subset A$ is retracted such that $C_{L}$ becomes unit in propagation. $C_{L}$ is called an asserting clause (cube) 14. Clauses (cubes) derived by rules cl-init and gen-cl-init cu-init and gen-cu-init) are used in exactly the same way in learning to produce asserting clauses (cubes).

QCDCL terminates ("UNSAT" or "SAT" in Fig. 1) by deriving the empty learned clause (cube) $C_{L}=\emptyset$. A clause (cube) resolution proof of the unsatisfiability (satisfiability) of $\psi$ can be obtained from the derivations of the learned clauses (cubes) up to the empty clause (cube).

By applying the generalized axioms using a complete QBF decision procedure, the empty assignment $A$, and an unlimited amount of time, the empty clause (cube) can be derived right away from any given unsatisfiable (satisfiable) PCNF $\psi$. In practice it is crucial to apply incomplete polynomial time procedures to limit the time spent on the satisfiability checks. However, the costs of frequent checks may outweigh the benefits. Hence in DepQBF 6.0, satisfiability checks for 
applications of the generalized axioms are dynamically disabled if they turn out to be too costly, and the traditional axioms are used instead. We refer to related work for implementation details 25 31].

\section{Features of DepQBF}

We briefly summarize the general features of DepQBF that have been incorporated since its initial version 0.1 [2627]. Most features were described in related publications. Additionally, we comment on the compatibility of the features with the implementation of QRES with generalized axioms (Fig. 1) in DepQBF 6.0.

Dependency Schemes. Since the initial version 0.1, DepQBF has been equipped with the standard dependency scheme 41 to relax the linear quantifier ordering in the prefix of a given PCNF $\psi$. In general, dependency schemes are used to compute dependency relations $D$, which are binary relations over the set of variables in $\psi$. If $(x, y) \notin D$ for two variables $x$ and $y$ then the ordering of $x$ and $y$ in $\psi$ can safely be swapped. Otherwise, if $(x, y) \in D$ then $y$ is considered to depend on $x$. The integration of dependency schemes in QCDCL results in the following reduction rule, which is added to QRES and implemented in DepQBF.

Definition 7 (Dependency-Aware Reduction [27]). Let $\psi=\Pi$. $\phi$ be $a$ $P C N F$ and $D$ be a dependency relation computed using a dependency scheme.

$$
\begin{aligned}
& \text { (1) } C \text { is a clause, } \mathrm{Q}(\Pi, l)=\forall \text {, } \\
& \frac{C \cup\{l\}}{C} \quad \begin{array}{ll}
\left(l, l^{\prime}\right) \notin D \text { for all } l^{\prime} \in C \text { with } \mathrm{Q}\left(\Pi, l^{\prime}\right)=\exists \text { or } \quad \text { (2) } C \text { is a cube, } \mathrm{Q}(\Pi, l)=\exists, &
\end{array} \\
& \left(l, l^{\prime}\right) \notin D \text { for all } l^{\prime} \in C \text { with } \mathrm{Q}\left(\Pi, l^{\prime}\right)=\forall
\end{aligned}
$$

Rule dep-red generalizes the traditional reduction rule red by the use of dependency relation instead of the linear ordering of variables $\left(\leq_{\Pi}\right)$ in the prefix of PCNF $\psi$. This way, it might be possible to reduce literals by rule dep-red which cannot be reduced by rule red. The soundness of QRES with rule dep-red has been proved for a dependency relation that is even more general (and thus allows for additional reductions) than the one implemented in DepQBF 444546 . The generalized axioms gen-cl-init and gen-cu-init of QRES implemented in DepQBF 6.0 are naturally compatible with rule dep-red. Additionally, dependency schemes enable a relaxed variant of QCDCL assignments (Definition 2 based on the respective dependency relation rather than the prefix ordering of a PCNF $\psi$.

Long-Distance Resolution. The Q-resolution rule res 21] explicitly disallows to generate clauses (cubes) that are tautological (contradictory). This restriction is relaxed under certain side conditions in long-distance (LD) Q-resolution 24849. LDQ-resolution was first implemented in the QCDCL solver Quaffle [48] and was incorporated in version 3.0 of DepQBF. Compared to QRES with traditional Q-resolution res 21, QRES with LDQ-resolution is exponentially more powerful in terms of proof sizes [13]. The generalized axioms gen-cl-init and gen-cu-init 
implemented in DepQBF 6.0 are not only compatible with the LDQ-resolution rule, but with any variants of Q-resolution (cf. [3). Recently, the soundness of the combination of LDQ-resolution of clauses and dependency schemes in QRES has been proved 4 437, leaving the soundness of cube resolutions as an open problem. Therefore, the combination of LDQ-resolution and dependency schemes is not supported in DepQBF 6.0.

Incremental Solving. Since version 3.0, DepQBF has been equipped with an API in C and Java for incremental solving of sequences $S:=\left\langle\psi_{0}, \ldots, \psi_{n}\right\rangle$ of syntactically related PCNFs $\psi_{i}$ [28/34. Incremental solving aims at reusing the clauses and cubes that were learned when solving PCNF $\psi_{i}$ when it comes to solve the PCNFs $\psi_{j}$ with $i<j$. The API of DepQBF allows to modify the PCNFs in $S$ by manipulating the quantifier prefix and adding or removing sets of clauses in a stack-based way. Since version 4.0, it is possible to add or remove sets of clauses arbitrarily [29] and to extract unsatisfiable cores, i.e., unsatisfiable subformulas of the PCNF $\psi_{i}$. At any time when solving $\psi_{i} \in S$, the soundness property of QCDCL (Section 3) that $\psi \equiv_{\text {sat }} \psi_{\theta}$ and $\psi \equiv_{\text {sat }} \psi_{\gamma}$, where $\psi=\psi_{i}$, must hold. To guarantee that property when using the generalized axioms for incremental solving, DepQBF 6.0 currently only applies the generalized cube axiom gen-cu-init with dynamic QBCE used to check satisfiability of $\psi_{\gamma}^{\prime}$ in conflict/solution detection (Fig. 11. Although this configuration restricts the power of the generalized axioms, it has improved incremental solving in the context of QBF-based conformant planning [12. As it is unclear how to use dependency schemes effectively in incremental solving, their application is disabled in DepQBF 6.0.

Generation of Proofs and Certificates. QCDCL solvers can produce clause (cube) resolution proofs of the unsatisfiability (satisfiability) of PCNFs as a byproduct of clause (cube) learning. Since version 1.0 [36, DepQBF is capable of producing proofs without employing dependency schemes by rule dep-red Given a proof $P$ of a PCNF $\psi$, a certificate of $\psi$ can be extracted from $P$ by inspecting the reduction steps by rule $r e d$ in $P$ [2]. A certificate of an unsatisfiable (satisfiable) PCNF $\psi$ is given by a set of Herbrand (Skolem) functions which represent the universal (existential) variables in $\psi$. Applications of the generalized axioms in QCDCL in general impose considerable restrictions on the certificate extraction process. The workflow 2 to extract a certificate from $P$ was originally presented for traditional QRES proofs. If proof $P$ contains clauses (cubes) derived by rule gen-cl-init (rule gen-cu-init), then $P$ may lack information needed to extract correct certificates. As a result, DepQBF 6.0 does not support cube resolution proof generation combined with the generalized cube axiom gen-cu-init However, it supports clause resolution proof generation with the generalized clause axiom gen-cl-init provided that only propositional abstractions and SAT solving are used for satisfiability checking in the side condition of this axiom.

Advanced Generation of Learned Clauses and Cubes. The derivation of a single asserting clause (cube) starting from a falsified clause (satisfied cube) as implemented in traditional QCDCL [1424/48] has an exponential worst case [47]. 
Table 1. Solved instances $(S)$, solved unsatisfiable $(\perp)$ and satisfiable ones $(\top)$, uniquely solved ones among all solvers $(U)$, and total wall clock time including time outs on 825 PCNFs from QBFEVAL'16 without (1a) and with preprocessing by Bloqqer (1b).

(a) Original instances.

\begin{tabular}{lcccrc}
\hline Solver & $S$ & $\perp$ & \multicolumn{1}{c}{ T } & U & Time \\
\hline AIGSolve & 603 & 301 & 302 & 34 & $440 \mathrm{~K}$ \\
GhostQ & 593 & 292 & 301 & 7 & $457 \mathrm{~K}$ \\
QSTS & 578 & 294 & 284 & 3 & $469 \mathrm{~K}$ \\
DQ & 458 & 255 & 203 & 0 & $682 \mathrm{~K}$ \\
DQ-linldq & 458 & 257 & 201 & 2 & $686 \mathrm{~K}$ \\
DQ-lin & 456 & 255 & 201 & 0 & $686 \mathrm{~K}$ \\
DQ-ncl & 448 & 246 & 202 & 0 & $703 \mathrm{~K}$ \\
DQ-nq & 397 & 228 & 169 & 0 & $788 \mathrm{~K}$ \\
DQ-ncu & 393 & 229 & 164 & 0 & $796 \mathrm{~K}$ \\
DQ-n & 383 & 221 & 162 & 0 & $814 \mathrm{~K}$ \\
CAQE & 378 & 202 & 176 & 9 & $831 \mathrm{~K}$ \\
QESTO & 369 & 210 & 159 & 0 & $864 \mathrm{~K}$ \\
RAReQS & 341 & 211 & 130 & 2 & $891 \mathrm{~K}$ \\
\hline
\end{tabular}

(b) Preprocessed by Bloqqer.

\begin{tabular}{lcccrc}
\hline Solver & $S$ & $\perp$ & $T$ & $U$ & Time \\
\hline QSTS & 633 & 330 & 303 & 11 & $365 \mathrm{~K}$ \\
RAReQS & 633 & 334 & 299 & 8 & $375 \mathrm{~K}$ \\
QESTO & 620 & 321 & 299 & 0 & $395 \mathrm{~K}$ \\
DQ-ncl & 601 & 303 & 298 & 0 & $428 \mathrm{~K}$ \\
DQ & 601 & 301 & 300 & 0 & $429 \mathrm{~K}$ \\
DQ-linldq & 598 & 300 & 298 & 2 & $437 \mathrm{~K}$ \\
DQ-lin & 597 & 299 & 298 & 0 & $436 \mathrm{~K}$ \\
CAQE & 596 & 301 & 295 & 4 & $451 \mathrm{~K}$ \\
DQ-n & 593 & 296 & 297 & 0 & $444 \mathrm{~K}$ \\
DQ-ncu & 591 & 297 & 294 & 0 & $455 \mathrm{~K}$ \\
DQ-nq & 587 & 293 & 294 & 0 & $455 \mathrm{~K}$ \\
GhostQ & 570 & 282 & 288 & 0 & $485 \mathrm{~K}$ \\
AIGSolve & 567 & 286 & 281 & 14 & $481 \mathrm{~K}$ \\
\hline
\end{tabular}

Since version 2.0 DepQBF comes with an approach that avoids this exponential case 32 by a revised selection of clauses (cubes) to be resolved in learning. This advanced approach is compatible with all the techniques presented above.

\section{Experiments}

We compare variants of DepQBF 6.02, which is the latest follow-up release of DepQBF 6.0, to top performing solvers of QBFEVAL'16 38. As benchmarks we consider all 825 instances from the PCNF track, both in original form (Table 1a) and preprocessed by Bloqqer version 37 (Table 1b). We take preprocessing into account as it might have a positive impact on certain solvers while a negative on others (cf. 33 35]). Experiments were run on an AMD Opteron 6238 processor $(2.6 \mathrm{GHz})$ under 64-bit Ubuntu Linux 12.04 with time and memory limits of 1800 seconds and seven GB. Exceeding the memory limit is counted as a time out 3

To assess the impact of the generalized axioms gen-cl-init and gen-cu-init on the performance, we consider DepQBF 6.02 using both gen-cl-init and gen-cu-init (variant $\mathrm{DQ}$ in the tables), without gen-cl-init (DQ-ncl), without gen-cu-init (DQ-ncu), and using no generalized axioms at all (DQ-n).

On original instances (Table 1a), DQ outperforms variants DQ-ncl, DQ-ncu, and DQ-n with restricted or without generalized axioms, respectively. Variant DQncl without axiom gen-cl-init outperforms variant DQ-ncu without gen-cu-init We attribute this effect to the use of dynamic QBCE (among other techniques) for applications of the cube axiom gen-cu-init in DQ-ncl. Compared to DQ, disabling only dynamic QBCE in variant DQ-nq severely impacts performance.

\footnotetext{
${ }^{3}$ We refer to the appendix of this paper with additional experimental results.
} 
Table 2. Related to Table 1a solver performance on 402 filtered original (not preprocessed) instances partitioned into 261 instances with at most two 2a and 141 with three or more quantifier alternations $2 \mathrm{~b}$.

(a) At most two quantifier alternations.

\begin{tabular}{lrrrrr}
\hline Solver & \multicolumn{1}{c}{$S$} & $\perp$ & \multicolumn{1}{c}{ T } & $U$ & Time \\
\hline GhostQ & 176 & 75 & 101 & 5 & $171 \mathrm{~K}$ \\
AIGSolve & 138 & 66 & 72 & 14 & $250 \mathrm{~K}$ \\
QSTS & 136 & 58 & 78 & 0 & $232 \mathrm{~K}$ \\
RAReQS & 76 & 43 & 33 & 1 & $340 \mathrm{~K}$ \\
DQ-lin & 69 & 35 & 34 & 0 & $351 \mathrm{~K}$ \\
DQ & 69 & 35 & 34 & 0 & $351 \mathrm{~K}$ \\
DQ-ncl & 68 & 35 & 33 & 0 & $354 \mathrm{~K}$ \\
DQ-linldq & 67 & 34 & 33 & 0 & $354 \mathrm{~K}$ \\
QESTO & 66 & 37 & 29 & 0 & $359 \mathrm{~K}$ \\
DQ-ncu & 53 & 24 & 29 & 0 & $378 \mathrm{~K}$ \\
DQ-n & 52 & 24 & 28 & 0 & $378 \mathrm{~K}$ \\
DQ-nq & 52 & 23 & 29 & 0 & $379 \mathrm{~K}$ \\
CAQE & 43 & 17 & 26 & 3 & $397 \mathrm{~K}$ \\
\hline
\end{tabular}

(b) Three or more quantifier alternations.

\begin{tabular}{lccccc}
\hline Solver & $S$ & $\perp$ & $T$ & $U$ & Time \\
\hline DQ-linldq & 81 & 50 & 31 & 2 & $120 \mathrm{~K}$ \\
DQ & 79 & 47 & 32 & 0 & $119 \mathrm{~K}$ \\
DQ-ncl & 79 & 47 & 32 & 0 & $120 \mathrm{~K}$ \\
DQ-lin & 78 & 47 & 31 & 0 & $123 \mathrm{~K}$ \\
QSTS & 72 & 44 & 28 & 3 & $132 \mathrm{~K}$ \\
DQ-nq & 56 & 37 & 19 & 0 & $159 \mathrm{~K}$ \\
GhostQ & 56 & 31 & 25 & 2 & $160 \mathrm{~K}$ \\
DQ-n & 55 & 36 & 19 & 0 & $159 \mathrm{~K}$ \\
DQ-ncu & 55 & 36 & 19 & 0 & $159 \mathrm{~K}$ \\
AIGSolve & 54 & 25 & 29 & 9 & $161 \mathrm{~K}$ \\
QESTO & 49 & 33 & 16 & 0 & $179 \mathrm{~K}$ \\
CAQE & 46 & 29 & 17 & 2 & $182 \mathrm{~K}$ \\
RAReQS & 43 & 33 & 10 & 0 & $180 \mathrm{~K}$ \\
\hline
\end{tabular}

On preprocessed instances (Table $1 \mathrm{~b}$ ), we make similar observations regarding the impact of the generalized axioms like in Table 1a. However, variant DQncl without the clause axiom gen-cl-init is on par with DQ. Preprocessing may blur the structure of an instance. We conjecture that this blurring hinders the success of the QBF decision procedures in DepQBF, on which applications of the generalized axioms are based. In general the performance difference between the variants of DepQBF is smaller than on original instances. The rankings of the solvers RAReQS [17], QESTO [19], and CAQE [39] are improved substantially by preprocessing, whereas those of AIGSolve [42] and GhostQ [17/22] become worse. The best variant DQ-ncl in Table 1b ranks fourth behind QSTS [7, RAReQS, and QESTO. However, the lag to the solver ranked third is 19 instances compared to 120 instances for the best variant DQ in Table 1 a that also ranks fourth.

To analyze the effects of preprocessing in more detail, we filtered the 825 PCNFs from QBFEVAL'16 by discarding 354 PCNFs that are already solved by Bloqqer and 69 PCNFs where Bloqqer eliminated all universally quantified variables, resulting in a set of 402 PCNFs. Further, we considered the 402 PCNFs in their original form and preprocessed by Bloqqer and partitioned them into subsets containing PCNFs with at most two and with three or more quantifier alternations. Such partitioning is motivated by a related experimental study [30] where a large diversity of solver performance was observed on instance classes defined by alternations. Tables 2 and 3 show solver performance on these subsets without and with preprocessing, respectively. Notably, variants of DepQBF outperform the other solvers on the subsets with three or more alternations, both without and with preprocessing (Tables $2 \mathrm{~b}$ and $3 \mathrm{~b}$ ).

All variants of DepQBF reported above apply dependency-aware reduction by rule dep-red. Variant DQ-lin is the same as DQ (including generalized axioms) 
Table 3. Related to Table $1 \mathrm{~b}$ solver performance on 402 filtered and preprocessed instances partitioned into 270 instances with at most two $3 \mathrm{a}$ and 132 with three or more quantifier alternations $3 \mathrm{~b}$.

(a) At most two quantifier alternations.

\begin{tabular}{lccccc}
\hline Solver & $S$ & $\perp$ & $\top$ & $U$ & Time \\
\hline RAReQS & 157 & 79 & 78 & 8 & $227 \mathrm{~K}$ \\
QESTO & 138 & 66 & 72 & 0 & $255 \mathrm{~K}$ \\
QSTS & 136 & 62 & 74 & 2 & $255 \mathrm{~K}$ \\
CAQE & 118 & 49 & 69 & 2 & $298 \mathrm{~K}$ \\
GhostQ & 111 & 46 & 65 & 1 & $304 \mathrm{~K}$ \\
DQ & 107 & 43 & 64 & 1 & $311 \mathrm{~K}$ \\
DQ-lin & 106 & 42 & 64 & 0 & $311 \mathrm{~K}$ \\
DQ-ncl & 105 & 43 & 62 & 0 & $312 \mathrm{~K}$ \\
DQ-n & 105 & 41 & 64 & 0 & $313 \mathrm{~K}$ \\
DQ-linldq & 104 & 40 & 64 & 0 & $315 \mathrm{~K}$ \\
AIGSolve & 102 & 49 & 53 & 7 & $313 \mathrm{~K}$ \\
DQ-nq & 102 & 39 & 63 & 0 & $322 \mathrm{~K}$ \\
DQ-ncu & 102 & 40 & 62 & 0 & $323 \mathrm{~K}$ \\
\hline
\end{tabular}

(b) Three or more quantifier alternations.

\begin{tabular}{lccccr}
\hline Solver & $S$ & $\perp$ & $\top$ & $U$ & Time \\
\hline DQ-ncl & 83 & 51 & 32 & 0 & $96 \mathrm{~K}$ \\
DQ & 81 & 49 & 32 & 0 & $98 \mathrm{~K}$ \\
DQ-linldq & 81 & 51 & 30 & 2 & $102 \mathrm{~K}$ \\
DQ-lin & 78 & 48 & 30 & 0 & $105 \mathrm{~K}$ \\
DQ-ncu & 76 & 48 & 28 & 0 & $112 \mathrm{~K}$ \\
QSTS & 75 & 50 & 25 & 1 & $107 \mathrm{~K}$ \\
DQ-n & 75 & 46 & 29 & 0 & $112 \mathrm{~K}$ \\
DQ-nq & 72 & 45 & 27 & 0 & $113 \mathrm{~K}$ \\
QESTO & 69 & 45 & 24 & 0 & $120 \mathrm{~K}$ \\
CAQE & 64 & 42 & 22 & 0 & $136 \mathrm{~K}$ \\
RAReQS & 62 & 45 & 17 & 1 & $131 \mathrm{~K}$ \\
AIGSolve & 51 & 27 & 24 & 6 & $151 \mathrm{~K}$ \\
GhostQ & 46 & 26 & 20 & 0 & $162 \mathrm{~K}$ \\
\hline
\end{tabular}

but uses the traditional reduction rule red based on the linear quantifier ordering of PCNFs. Variant DQ outperforms DQ-lin in all tables except Table 2a, where DQ-lin is on par, which illustrates the benefits of dependency schemes in QCDCL. Variant DQ-linldq differs from DQ-lin in the use of LDQ-resolution in learning instead of traditional Q-resolution by rule res. The results with LDQ-resolution are mixed, despite being a stronger proof system than Q-resolution. Variant DQlinldq outperforms DQ-lin in all tables except Tables $2 \mathrm{a}$ and $3 \mathrm{a}$, i.e., on instances with at most two quantifier alternations.

\section{Conclusion}

We presented the latest major release version 6.0 of the QCDCL solver DepQBF. DepQBF 6.0 implements a variant of QCDCL that is based on a generalization of the Q-resolution calculus (QRES). The generalization is achieved by equipping QRES with generalized clause and cube axioms to be used in clause and cube learning [31. The generalized axioms provide an extensible framework of interfaces for the integration of arbitrary QBF proof systems in QRES, and hence in QCDCL. The integration of proof systems orthogonal to Q-resolution, such as variable expansion, enables QCDCL to potentially produce proofs that are exponentially shorter than proofs produced by traditional QCDCL. This way, the state of the art of QCDCL solving can be further advanced. A related open problem is the inability of plain QCDCL to exploit the full power of Q-resolution [16.

The workflow of QCDCL with generalized axioms is not tailored towards DepQBF 6.0 but can be implemented in any QCDCL solver. Furthermore, it is compatible with dependency schemes [4145] and any Q-resolution variant [3, which offers potential for further improvements. 
Experiments with variants of DepQBF 6.0 showed considerable performance gains due to the application of generalized axioms. However, frequent applications are hindered by computationally expensive QBF satisfiability checks in the side conditions of the axioms. To limit the checking overhead, axiom applications must be carefully scheduled. In this respect, there is room for improvements in fine tuning DepQBF 6.0. Further, it may be beneficial to integrate the QBF decision procedures that are applied to satisfiability checking more tightly in the QCDCL workflow, like with dynamic blocked clause elimination (QBCE) 25].

\section{References}

1. Ayari, A., Basin, D.A.: QUBOS: Deciding Quantified Boolean Logic Using Propositional Satisfiability Solvers. In: FMCAD. LNCS, vol. 2517, pp. 187-201. Springer (2002)

2. Balabanov, V., Jiang, J.R.: Unified QBF Certification and its Applications. Formal Methods in System Design 41(1), 45-65 (2012)

3. Balabanov, V., Widl, M., Jiang, J.R.: QBF Resolution Systems and Their Proof Complexities. In: SAT. LNCS, vol. 8561, pp. 154-169. Springer (2014)

4. Beyersdorff, O., Blinkhorn, J.: Dependency Schemes in QBF Calculi: Semantics and Soundness. In: CP. LNCS, vol. 9892, pp. 96-112. Springer (2016)

5. Beyersdorff, O., Chew, L., Janota, M.: Proof Complexity of Resolution-based QBF Calculi. In: STACS. LIPIcs, vol. 30, pp. 76-89. Schloss Dagstuhl-Leibniz-Zentrum fuer Informatik (2015)

6. Biere, A.: Resolve and Expand. In: SAT. LNCS, vol. 3542, pp. 59-70. Springer (2004)

7. Bogaerts, B., Janhunen, T., Tasharrofi, S.: SAT-to-SAT in QBFEval 2016. In: QBF Workshop. CEUR Workshop Proceedings, vol. 1719, pp. 63-70. CEUR-WS.org (2016)

8. Bubeck, U., Kleine Büning, H.: Bounded Universal Expansion for Preprocessing QBF. In: SAT. LNCS, vol. 4501, pp. 244-257. Springer (2007)

9. Cadoli, M., Giovanardi, A., Schaerf, M.: An Algorithm to Evaluate Quantified Boolean Formulae. In: AAAI. pp. 262-267. AAAI Press / The MIT Press (1998)

10. Clarke, E.M., Grumberg, O., Jha, S., Lu, Y., Veith, H.: Counterexample-Guided Abstraction Refinement for Symbolic Model Checking. J. ACM 50(5), 752-794 (2003)

11. Davis, M., Logemann, G., Loveland, D.W.: A Machine Program for TheoremProving. Commun. ACM 5(7), 394-397 (1962)

12. Egly, U., Kronegger, M., Lonsing, F., Pfandler, A.: Conformant Planning as a Case Study of Incremental QBF Solving. Ann. Math. Artif. Intell. 80(1), 21-45 (2017)

13. Egly, U., Lonsing, F., Widl, M.: Long-Distance Resolution: Proof Generation and Strategy Extraction in Search-Based QBF Solving. In: LPAR. LNCS, vol. 8312, pp. 291-308. Springer (2013)

14. Giunchiglia, E., Narizzano, M., Tacchella, A.: Clause/Term Resolution and Learning in the Evaluation of Quantified Boolean Formulas. JAIR 26, 371-416 (2006)

15. Heule, M., Järvisalo, M., Lonsing, F., Seidl, M., Biere, A.: Clause Elimination for SAT and QSAT. JAIR 53, 127-168 (2015)

16. Janota, M.: On Q-Resolution and CDCL QBF Solving. In: SAT. LNCS, vol. 9710, pp. 402-418. Springer (2016) 
17. Janota, M., Klieber, W., Marques-Silva, J., Clarke, E.: Solving QBF with Counterexample Guided Refinement. Artif. Intell. 234, 1-25 (2016)

18. Janota, M., Marques-Silva, J.: Expansion-Based QBF Solving versus Q-Resolution. Theor. Comput. Sci. 577, 25-42 (2015)

19. Janota, M., Marques-Silva, J.: Solving QBF by Clause Selection. In: IJCAI. pp. 325-331. AAAI Press (2015)

20. Kleine Büning, H., Bubeck, U.: Theory of Quantified Boolean Formulas. In: Handbook of Satisfiability, FAIA, vol. 185, pp. 735-760. IOS Press (2009)

21. Kleine Büning, H., Karpinski, M., Flögel, A.: Resolution for Quantified Boolean Formulas. Inf. Comput. 117(1), 12-18 (1995)

22. Klieber, W., Sapra, S., Gao, S., Clarke, E.M.: A Non-Prenex, Non-Clausal QBF Solver with Game-State Learning. In: SAT. LNCS, vol. 6175, pp. 128-142. Springer (2010)

23. Kullmann, O.: On a Generalization of Extended Resolution. Discrete Applied Mathematics 96-97, 149-176 (1999)

24. Letz, R.: Lemma and Model Caching in Decision Procedures for Quantified Boolean Formulas. In: TABLEAUX. LNCS, vol. 2381, pp. 160-175. Springer (2002)

25. Lonsing, F., Bacchus, F., Biere, A., Egly, U., Seidl, M.: Enhancing Search-Based QBF Solving by Dynamic Blocked Clause Elimination. In: LPAR. LNCS, vol. 9450, pp. 418-433. Springer (2015)

26. Lonsing, F., Biere, A.: DepQBF: A Dependency-Aware QBF Solver. JSAT 7(2-3), 71-76 (2010)

27. Lonsing, F., Biere, A.: Integrating Dependency Schemes in Search-Based QBF Solvers. In: SAT. LNCS, vol. 6175, pp. 158-171. Springer (2010)

28. Lonsing, F., Egly, U.: Incremental QBF Solving. In: CP. LNCS, vol. 8656, pp. 514-530. Springer (2014)

29. Lonsing, F., Egly, U.: Incrementally Computing Minimal Unsatisfiable Cores of QBFs via a Clause Group Solver API. In: SAT. LNCS, vol. 9340, pp. 191-198. Springer (2015)

30. Lonsing, F., Egly, U.: Evaluating QBF Solvers: Quantifier Alternations Matter. CoRR abs/1701.06612 (2017), http://arxiv.org/abs/1701.06612, technical report

31. Lonsing, F., Egly, U., Seidl, M.: Q-Resolution with Generalized Axioms. In: SAT. LNCS, vol. 9710, pp. 435-452. Springer (2016)

32. Lonsing, F., Egly, U., Van Gelder, A.: Efficient Clause Learning for Quantified Boolean Formulas via QBF Pseudo Unit Propagation. In: SAT. LNCS, vol. 7962, pp. 100-115. Springer (2013)

33. Lonsing, F., Seidl, M., Van Gelder, A.: The QBF Gallery: Behind the Scenes. Artif. Intell. 237, 92-114 (2016)

34. Marin, P., Miller, C., Lewis, M.D.T., Becker, B.: Verification of Partial Designs Using Incremental QBF Solving. In: DATE. pp. 623-628. IEEE (2012)

35. Marin, P., Narizzano, M., Pulina, L., Tacchella, A., Giunchiglia, E.: Twelve Years of QBF Evaluations: QSAT Is PSPACE-Hard and It Shows. Fundam. Inform. 149(1-2), 133-158 (2016)

36. Niemetz, A., Preiner, M., Lonsing, F., Seidl, M., Biere, A.: Resolution-Based Certificate Extraction for QBF - (Tool Presentation). In: SAT. LNCS, vol. 7317, pp. 430-435. Springer (2012)

37. Peitl, T., Slivovsky, F., Szeider, S.: Long Distance Q-Resolution with Dependency Schemes. In: SAT. LNCS, vol. 9710, pp. 500-518. Springer (2016) 
38. Pulina, L.: The Ninth QBF Solvers Evaluation - Preliminary Report. In: Proceedings of the 4th International Workshop on Quantified Boolean Formulas QBF 2016. CEUR Workshop Proceedings, vol. 1719, pp. 1-13. CEUR-WS.org (2016)

39. Rabe, M.N., Tentrup, L.: CAQE: A Certifying QBF Solver. In: FMCAD. pp. 136-143. IEEE (2015)

40. Robinson, J.A.: A Machine-Oriented Logic Based on the Resolution Principle. J. ACM 12(1), 23-41 (1965)

41. Samer, M., Szeider, S.: Backdoor Sets of Quantified Boolean Formulas. JAR 42(1), 77-97 (2009)

42. Scholl, C., Pigorsch, F.: The QBF Solver AIGSolve. In: QBF Workshop. CEUR Workshop Proceedings, vol. 1719, pp. 55-62. CEUR-WS.org (2016)

43. Silva, J.P.M., Lynce, I., Malik, S.: Conflict-Driven Clause Learning SAT Solvers. In: Handbook of Satisfiability, FAIA, vol. 185, pp. 131-153. IOS Press (2009)

44. Slivovsky, F., Szeider, S.: Computing Resolution-Path Dependencies in Linear Time. In: SAT. LNCS, vol. 7317, pp. 58-71. Springer (2012)

45. Slivovsky, F., Szeider, S.: Soundness of Q-Resolution with Dependency Schemes. Theor. Comput. Sci. 612, 83-101 (2016)

46. Van Gelder, A.: Variable Independence and Resolution Paths for Quantified Boolean Formulas. In: CP. LNCS, vol. 6876, pp. 789-803. Springer (2011)

47. Van Gelder, A.: Contributions to the Theory of Practical Quantified Boolean Formula Solving. In: CP. LNCS, vol. 7514, pp. 647-663. Springer (2012)

48. Zhang, L., Malik, S.: Conflict Driven Learning in a Quantified Boolean Satisfiability Solver. In: ICCAD. pp. 442-449. ACM / IEEE Computer Society (2002)

49. Zhang, L., Malik, S.: Towards a Symmetric Treatment of Satisfaction and Conflicts in Quantified Boolean Formula Evaluation. In: CP. LNCS, vol. 2470, pp. 200-215. Springer (2002) 


\section{A Additional Experimental Data}

Table 4. Related to Table 1a (no preprocessing): the 825 PCNFs are partitioned into 466 PCNFs with at most two (4a), and 359 PCNFs with three or more quantifier alternations $4 \mathrm{~b}$.

(a) At most two quantifier alternations. (b) Three or more quantifier alternations.

\begin{tabular}{|c|c|c|c|c|c|c|c|c|c|c|c|}
\hline Solver & $S$ & $\perp$ & $T$ & $U$ & Time & Solver & $S$ & $\perp$ & $T$ & $U$ & Time \\
\hline AIGSolve & 338 & 170 & 168 & 25 & $262 \mathrm{~K}$ & QSTS & 286 & 151 & 135 & 3 & $144 \mathrm{~K}$ \\
\hline GhostQ & 336 & 160 & 176 & 5 & $257 \mathrm{~K}$ & AIGSolve & 265 & 131 & 134 & 9 & $177 \mathrm{~K}$ \\
\hline QSTS & 292 & 143 & 149 & 0 & $325 \mathrm{~K}$ & GhostQ & 257 & 132 & 125 & 2 & $199 \mathrm{~K}$ \\
\hline DQ-lin & 233 & 126 & 107 & 0 & $429 \mathrm{~K}$ & DQ-linldq & 227 & 132 & 95 & 2 & $254 \mathrm{~K}$ \\
\hline $\mathrm{DQ}$ & 233 & 126 & 107 & 0 & $429 \mathrm{~K}$ & $\mathrm{DQ}$ & 225 & 129 & 96 & 0 & $253 \mathrm{~K}$ \\
\hline DQ-linldq & 231 & 125 & 106 & 0 & $431 \mathrm{~K}$ & DQ-ncl & 225 & 129 & 96 & 0 & $254 \mathrm{~K}$ \\
\hline RAReQS & 231 & 140 & 91 & 2 & $434 \mathrm{~K}$ & DQ-lin & 223 & 129 & 94 & 0 & $257 \mathrm{~K}$ \\
\hline DQ-ncl & 223 & 117 & 106 & 0 & $449 \mathrm{~K}$ & CAQE & 204 & 111 & 93 & 5 & $295 \mathrm{~K}$ \\
\hline QESTO & 213 & 126 & 87 & 0 & $474 \mathrm{~K}$ & DQ-ncu & 196 & 118 & 78 & 0 & $304 \mathrm{~K}$ \\
\hline DQ-nq & 202 & 110 & 92 & 0 & $483 \mathrm{~K}$ & DQ-n & 195 & 118 & 77 & 0 & $304 \mathrm{~K}$ \\
\hline DQ-ncu & 197 & 111 & 86 & 0 & $492 \mathrm{~K}$ & DQ-nq & 195 & 118 & 77 & 0 & $305 \mathrm{~K}$ \\
\hline DQ-n & 188 & 103 & 85 & 0 & $509 \mathrm{~K}$ & QESTO & 156 & 84 & 72 & 0 & $390 K$ \\
\hline CAQE & 174 & 91 & 83 & 4 & $535 \mathrm{~K}$ & RAReQS & 110 & 71 & 39 & 0 & $456 \mathrm{~K}$ \\
\hline
\end{tabular}


Table 5. Related to Table $1 \mathrm{~b}$ (preprocessed by Bloqqer): the 825 PCNFs are partitioned into 693 PCNFs with at most two (5a), and 132 PCNFs with three or more quantifier alternations $5 \mathrm{~b}$.

(a) At most two quantifier alternations.

(b) Three or more quantifier alternations.

\begin{tabular}{lcccrc}
\hline Solver & $S$ & $\perp$ & $\top$ & $U$ & Time \\
\hline RAReQS & 571 & 289 & 282 & 16 & $243 \mathrm{~K}$ \\
QSTS & 558 & 280 & 278 & 2 & $258 \mathrm{~K}$ \\
QESTO & 551 & 276 & 275 & 0 & $274 \mathrm{~K}$ \\
CAQE & 532 & 259 & 273 & 2 & $315 \mathrm{~K}$ \\
GhostQ & 524 & 256 & 268 & 1 & $323 \mathrm{~K}$ \\
DQ & 520 & 252 & 268 & 1 & $331 \mathrm{~K}$ \\
DQ-lin & 519 & 251 & 268 & 0 & $331 \mathrm{~K}$ \\
DQ-n & 518 & 250 & 268 & 0 & $332 \mathrm{~K}$ \\
DQ-ncl & 518 & 252 & 266 & 0 & $332 \mathrm{~K}$ \\
DQ-linldq & 517 & 249 & 268 & 0 & $334 \mathrm{~K}$ \\
AIGSolve & 516 & 259 & 257 & 7 & $329 \mathrm{~K}$ \\
DQ-nq & 515 & 248 & 267 & 0 & $342 \mathrm{~K}$ \\
DQ-ncu & 515 & 249 & 266 & 0 & $342 \mathrm{~K}$ \\
\hline
\end{tabular}

\begin{tabular}{lccccr}
\hline Solver & $S$ & $\perp$ & $\top$ & $U$ & Time \\
\hline DQ-ncl & 83 & 51 & 32 & 0 & $96 \mathrm{~K}$ \\
DQ & 81 & 49 & 32 & 0 & $98 \mathrm{~K}$ \\
DQ-linldq & 81 & 51 & 30 & 2 & $102 \mathrm{~K}$ \\
DQ-lin & 78 & 48 & 30 & 0 & $105 \mathrm{~K}$ \\
DQ-ncu & 76 & 48 & 28 & 0 & $112 \mathrm{~K}$ \\
QSTS & 75 & 50 & 25 & 1 & $107 \mathrm{~K}$ \\
DQ-n & 75 & 46 & 29 & 0 & $112 \mathrm{~K}$ \\
DQ-nq & 72 & 45 & 27 & 0 & $113 \mathrm{~K}$ \\
QESTO & 69 & 45 & 24 & 0 & $120 \mathrm{~K}$ \\
CAQE & 64 & 42 & 22 & 0 & $136 \mathrm{~K}$ \\
RAReQS & 62 & 45 & 17 & 1 & $131 \mathrm{~K}$ \\
AIGSolve & 51 & 27 & 24 & 6 & $151 \mathrm{~K}$ \\
GhostQ & 46 & 26 & 20 & 0 & $162 \mathrm{~K}$ \\
\hline
\end{tabular}

